Research Paper

\title{
TERT Core Promotor Mutations in Early-Onset Bladder Cancer
}

\author{
Johannes Giedl ${ }^{1}$, Anja Rogler ${ }^{1}$, Andreas Wild ${ }^{1}$, Marc-Oliver Riener ${ }^{1}$, Thomas Filbeck ${ }^{2}$, Maximilian Burger ${ }^{3}$, \\ Petra Rümmele ${ }^{1}$, Carolyn Hurst ${ }^{4}$, Margaret Knowles ${ }^{4}$, Arndt Hartmannn ${ }^{1}$, Ulrike Zinnall ${ }^{1,5^{*}}$, Robert \\ Stoehr ${ }^{* \bowtie}$ \\ 1. Institute of Pathology, University Hospital Erlangen, Friedrich-Alexander University Erlangen-Nuremberg, Erlangen, Germany; \\ 2. Department of Urology, University Hospital Erlangen, Friedrich-Alexander University Erlangen-Nuremberg, Erlangen, Germany; \\ 3. Department of Urology, Caritas St. Josef Medical Center, University of Regensburg, Regensburg, Germany; \\ 4. Section of Experimental Oncology, Leeds Institute of Cancer and Pathology, University of Leeds, St. James's University Hospital, Leeds, United Kingdom; \\ 5. Institute of Medical Systems Biology, Max Delbrück Center for Molecular Medicine in the Helmholtz Association, Berlin, Germany. \\ *both authors contributed equally to this study. \\ $\square$ Corresponding author: PD Dr. Dr. Robert Stoehr, Institute of Pathology, University Hospital, Friedrich-Alexander University Erlangen-Nuremberg, \\ Krankenhausstr. 8-10, D-91054 Erlangen, Germany. phone: +49 913185 43610, e-mail: robert.stoehr@uk-erlangen.de.
}

(1) Ivyspring International Publisher. Reproduction is permitted for personal, noncommercial use, provided that the article is in whole, unmodified, and properly cited. See http://ivyspring.com/terms for terms and conditions.

Received: 2016.01.23; Accepted: 2016.03.14; Published: 2016.05.07

\begin{abstract}
Activating mutations in the core promoter of the TERT gene have been described in many different tumor entities. In vitro models showed a two- to fourfold increase in transcriptional activity of the TERT promoter through creation of a consensus binding motif for Ets/TCF transcription factors caused by these mutations. TERT core promoter mutations are the most common mutations in bladder cancer with a frequency between $55.6 \%$ and $82.8 \%$ described so far, and are independent of stage and grade. Since limited data on molecular alterations of early-onset bladder tumors exists, we assessed the frequency of TERT core promoter mutations in early-onset bladder cancer. Two cohorts of bladder tumors (early-onset patient group; $n=144$ (age of onset of disease $\leq 45$ years); unselected, consecutive group; $\mathrm{n}=125$ ) were examined for TERT core promoter mutations. After microdissection and extraction of DNA the corresponding hotspot regions in the TERT core promoter were examined by Sanger-sequencing or a SNaPshot approach. A significantly lower frequency of TERT core promoter mutations was found in tumors from the early-onset cohort compared to the consecutive cohort $(57.6 \%$ vs. $84.8 \%, p<0.001)$. Among the early-onset cohort cases younger than the cohort's median age of 39 years at disease onset showed a significantly reduced number of TERT promoter mutations $(31 / 67,46,3 \%)$ than cases aged between 39 and 45 years $(52 / 77,67.5 \% ; p=0.012)$. This association was not found in the consecutive cases. Mutation status was independent of tumor stage and grade. We conclude that in tumors from early-onset bladder cancer patients TERT core promoter mutations are not as frequent as in bladder tumors from consecutive cases, but seem to play an important role there as well. In patients below 39 years of age TERT core promoter mutations are a more infrequent event, suggesting different mechanisms of tumorigenesis in these young patients.
\end{abstract}

Key words: bladder cancer, early-onset, TERT, mutation, sequencing.

\section{Introduction}

The TERT gene encodes the catalytic reverse transcriptase subunit of telomerase, a RNA-dependent polymerase (ribonucleoprotein complex) maintaining telomere length, and preventing replicative senescence and genomic instability. In stem cells telomerase activity is crucial for indefinite replication and the limitless replicative potential associated with increased telomerase activity is one of the six hallmarks of cancer [1].

Elements responsible for the regulation of TERT 
promoter activity are located in a core region that includes $330 \mathrm{bp}$ upstream of the ATG start-codon and $37 \mathrm{bp}$ of exon 2 of the TERT gene. Recently, activating TERT core promoter mutations were described in familial and sporadic melanoma [2], and since then mutations have also been reported in a multitude of sporadic tumors [3]. Mutational hotspots are located at positions -57, -124 and $-146 \mathrm{bp}$. Mutations at these locations lead to nucleotide changes predicted to create novel binding motifs for E-twenty-six (Ets) transcription factors. In addition, binding motifs for the ternary complex factors (TCFs, which are a subfamily of Ets transcription factors) Elk1 and Elk4 are also putatively generated by these alterations. Elk1 and Elk4 are downstream targets of BRAF and involved in regulating expression of many genes. Creation of these de novo Ets/TCF-binding motifs leads to an increase in TERT promoter activity and gene expression [2].

Several studies shed light on the frequency of TERT core promoter mutations in urothelial carcinoma of the bladder. TERT core promoter mutations were found with a frequency up to $>80 \%$ in bladder cancer [4] and are the most common mutations in bladder cancer reported to date. Interestingly, TERT mutations showed neither a correlation to tumor stage or tumor grade, nor to disease outcome, but were suggested as a useful urine biomarker in the follow-up of bladder cancer patients or for proving the urothelial origin in cancers of unknown primary [5-7]. In addition, also in rare histopathological subtypes of bladder cancer (e.g. inverted papilloma, small cell carcinoma) these mutations were found but with various frequencies $[8,9]$.

It is well known that exposure to exogenous noxae such as amines, cigarette smoke, drugs and radiation therapy is associated with the development of bladder cancer, which is typically a disease of older people with a

Figure 1: Representative images of histopathological specimens (magnification: $x 100)$. A: Papillary urothelial neoplasia of low malignant potential (PUNLMP), TERT: wildtype. B: papillary PTa LG tumor, TERT wildtype. C: papillary PTa LG tumor, TERT: mutation: -124 "G > A". D: inverted papilloma, TERT: wildtype. mean age of diagnosis being between 65 and 70 years of age. Only a small fraction (approx. 1.5\%) of bladder tumors develop before the age of 45 years [10]. These tumors represent an interesting cohort to study, since tumorigenesis in such "early-onset" bladder tumors might be via a different pathway, or they might reveal crucial molecular alterations responsible for the initiation of urothelial carcinogenesis in general. Since only very limited data regarding molecular alterations of early-onset bladder tumors exist, we thought it reasonable to assess the frequency of TERT core promoter mutations in a cohort of such tumors, as this might reveal further evidence corroborating a different tumorigenesis pathway.

\section{Methods}

\section{Tissue samples}

Archival formalin-fixed paraffin-embedded (FFPE) tumor material from 269 cases acquired through transurethral resection was used for the study (cohort 1: unselected cases: $n=125$, cohort 2 : early-onset-bladder cancer cases (age at time of diagnosis $\leq 45$ years): $n=144$ ). The tumors were classified and staged according to the WHO classification of bladder tumors [11] and the current AJCC/TNM-classification system [12]. Representative images of available histopathological specimens are shown in Figure 1.

Clinicopathological characteristics of the cases are shown on Table 1. Prior IRB approval (University Hospital Erlangen, Germany) was obtained for the study.

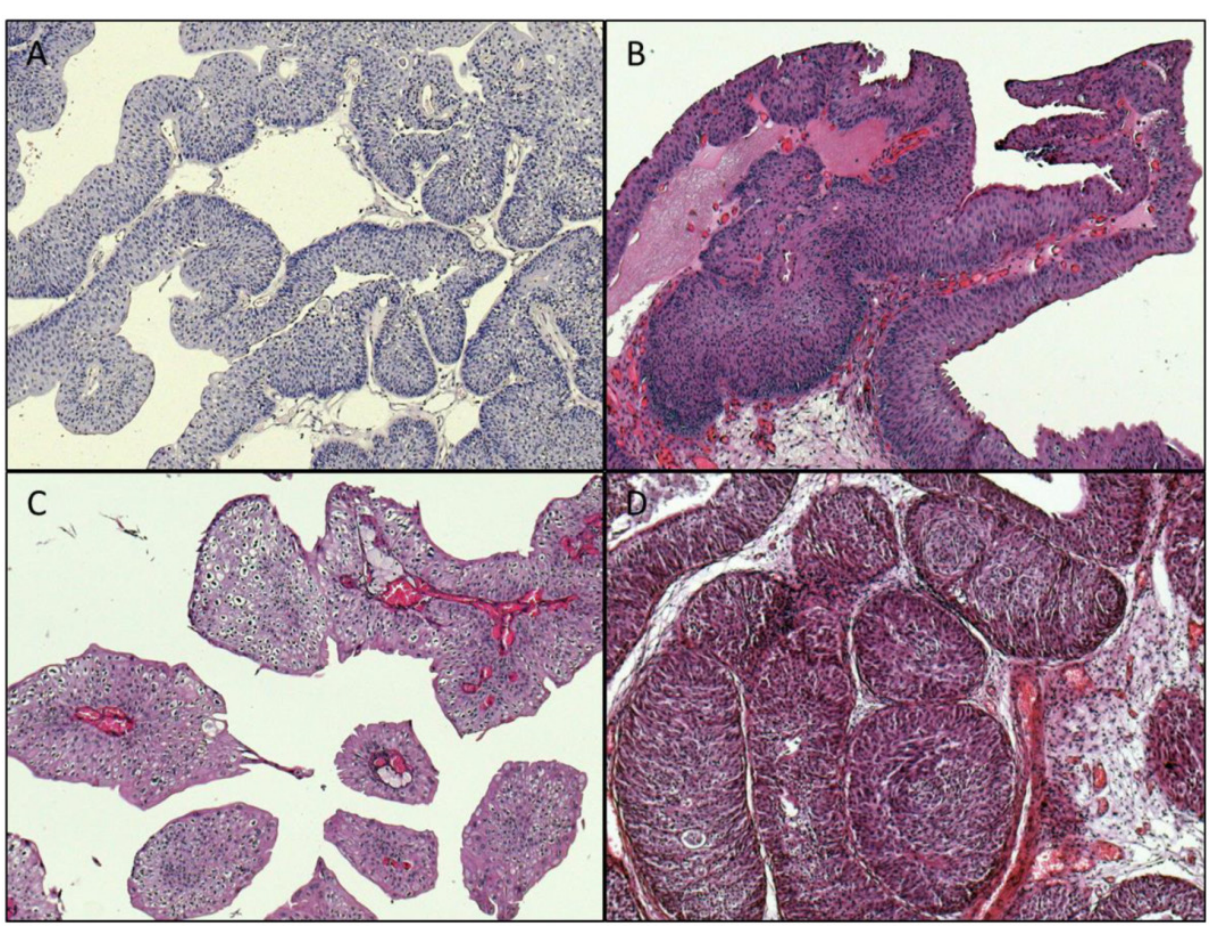


Table 1: Clinicopathological characteristics of the study cohorts.

\begin{tabular}{lllll}
\hline & \multicolumn{2}{l}{ Consecutive Cohort } & \multicolumn{2}{l}{ Early-Onset Tumor Cohort } \\
\hline Number & $\mathrm{n}=125$ & & $\mathrm{n}=144$ & \\
Age (years) & Median: 71 & Range: & Median: 39 & Range: \\
& Mean: 70.7 & $29-94$ & Mean: 36.6 & $11-45$ \\
Stage & Papilloma & $\mathrm{n}=2$ & & $\mathrm{n}=5$ \\
& Cis & $\mathrm{n}=1$ & & $\mathrm{n}=0$ \\
& Ta & $\mathrm{n}=78$ & & $\mathrm{n}=83$ \\
& T1 & $\mathrm{n}=35$ & & $\mathrm{n}=14$ \\
& $\geq$ T2 & $\mathrm{n}=8$ & & $\mathrm{n}=27$ \\
Grading & unknown & $\mathrm{n}=2$ & & $\mathrm{n}=16$ \\
& G1 & $\mathrm{n}=33$ & & $\mathrm{n}=43$ \\
& G2 & $\mathrm{n}=56$ & & $\mathrm{n}=48$ \\
& G3 & $\mathrm{n}=17$ & & $\mathrm{n}=30$ \\
Gender & unknown & $\mathrm{n}=19$ & & $\mathrm{n}=24$ \\
& Male & $\mathrm{n}=95$ & & $\mathrm{n}=100$ \\
& Female & $\mathrm{n}=30$ & & $\mathrm{n}=24$ \\
& unknown & $\mathrm{n}=0$ & & $\mathrm{n}=21$ \\
& & & &
\end{tabular}

\section{Microdissection and DNA isolation}

Microdissection and isolation of genomic DNA was carried out from FFPE tumor material as described previously [13]. In brief, $5 \mu \mathrm{m}$ thick serial sections of the tumor tissue were dewaxed and stained with $0.1 \%$ methylene blue for 15 seconds. Using an inverted microscope the tumor tissue (identified through matching with a marked H\&E-stained section reviewed by an experienced surgical pathologist) was scraped off with a sterile needle to obtain a tumor cell purity of at least $85 \%$. Isolation of genomic DNA from the microdissected tumor tissue was performed using the High Pure PCR Template Preparation Kit (Roche, Mannheim, Germany) according to the manufacturer's instructions.

\section{TERT promoter mutation analysis}

Mutation analysis of the TERT promoter was performed as described recently [2]. In brief, Sanger-sequencing of the TERT core promoter was carried out using an ABI Prism 3500 Genetic Analyzer and the Big Dye Terminator v.1.1 Cycle Sequencing Kit (Applied Biosystems, Foster City, Calif., USA) according to manufacturer's instructions. SNaPshot assays designed to detect hotspot mutations at positions -146, -124 and $-57 \mathrm{bp}$ of the TERT promoter were used when DNA samples were unsuitable for mutation analysis by Sanger sequencing. All primers and reaction conditions were described elsewhere in detail $[2,14]$.

\section{Statistics}

A two-sided Fisher's exact test was used to evaluate differences in the distribution of TERT promoter mutations according to stage, grade or age of the patients. Statistical analysis was carried out using SPSS version 13.0 (SPSS, Chicago, IL, USA). P values less than 0.05 were interpreted as statistically significant.

\section{Results}

All investigated cases were successfully analyzed by Sanger sequencing $(\mathrm{n}=16)$ or $\mathrm{SNaPshot}$ analysis $(n=253)$ (Figure 2). Overall, there was a significantly lower frequency of TERT promoter mutations in the tumors from the early-onset bladder cancer patients $(83 / 144,57.6 \%)$ compared to the unselected, consecutive cohort (106/125, 84.8\%; $\mathrm{p}<0.001$; Figure 3). The distribution of the detected TERT promoter mutations in our cohorts was very similar to the results of previously published bladder cancer studies $[4,5]$ with the majority of the mutated cases $(92 \%)$ carrying $-124 \mathrm{G}>\mathrm{A}(\mathrm{C}>\mathrm{T}$ on the opposite strand) or $-146 \mathrm{G}>\mathrm{A}$ mutations (Table 2). In order to find a possible age dependency of the occurrence of TERT promoter mutations in the tumors from the early-onset bladder cancer cohort we stratified this cohort against the median age (39 years) of the cohort. There was a significantly lower frequency of TERT promoter mutations in patients aged $<39$ years at time of first diagnoses compared to patients $\geq 39$ years $(\mathrm{p}=0.012$; Figure 4$)$. No further association was found between TERT promoter mutation and clinicopathological characteristics of the tumors.

Table 2: Distribution of TERT core promoter mutations in the two study cohorts.

\begin{tabular}{llll}
\hline $\begin{array}{l}\text { Distance from ATG } \\
\text { start site (bp) }\end{array}$ & Base change & $\begin{array}{l}\text { Consecutive } \\
\text { Cohort }(\mathrm{n}=125)\end{array}$ & $\begin{array}{l}\text { Early-Onset } \\
\text { Tumor Group } \\
(\mathrm{n}=144)\end{array}$ \\
\hline-57 & $\mathrm{~T}>\mathrm{G}$ & 6 & 0 \\
-124 & $\mathrm{G}>\mathrm{A}$ & 75 & 52 \\
-124 & $\mathrm{G}>\mathrm{T}$ & 2 & 1 \\
-146 & $\mathrm{G}>\mathrm{A}$ & 22 & 24 \\
-57 and -124 & $\mathrm{~T}>\mathrm{G}$ and G $>$ A & 1 & 0 \\
-124 and -146 & $\mathrm{G}>\mathrm{A}$ and G $>$ A & 0 & 5 \\
-124 and -146 & $\mathrm{G}>\mathrm{T}$ and $\mathrm{G}>\mathrm{A}$ & 0 & 1 \\
\hline
\end{tabular}

\section{Discussion}

In the present study we analysed TERT promoter mutations in a large cohort of tumors from patients with early-onset disease, and compared mutation data to that obtained from an unselected consecutive group of bladder tumors. Data from our consecutive cohort are in line with previously published studies [4-6] and argues for the reliability of the methods used and for a non-biased control group. Interestingly, there was a significant lower frequency of TERT promoter mutations in the tumors from the early-onset patients. This fact points to a possible 
age-dependent occurrence of TERT promoter mutations in bladder cancer or a different molecular background in early-onset bladder tumors. An age-dependent difference in TERT mutation frequency in bladder cancer was recently observed in two studies. $\mathrm{Wu}$ and coworkers found a significantly lower mutation frequency in tumors from patients aged younger than 50 years [6]. In this study tumors from patients below 50 years of age showed a mutation frequency of $37.5 \%$ vs $59.6 \%$ from patients $\geq 50$ years, however, only a small number of tumors from "younger" patients below 50 years of age $(n=40)$ was analysed and they did not justify why a cut-off of 50 years of age was selected. Despite these limitations data from $\mathrm{Wu}$ and colleagues are in line with the results from our study. Wang et al focused on TERT promotor mutation analysis of urothelial tumors from the upper urinary tract [15]. Within a cohort of ureter tumors the mean age of patients with mutated tumors was significantly higher than the mean age of patients with non-mutated tumors (72,4 years vs. 65,1 years). Although both cohorts showed a mean age typical for the regular onset of urothelial tumors the shift towards a lower TERT promoter frequency in an age-dependent manner is remarkable and also strengthens our findings. Interestingly, an age-dependent TERT promoter mutation frequency was also recently reported in papillary thyroid carcinoma. Liu and coworkers found a significant higher mutation frequency in patients aged $>45$ years compared to patients aged $\leq 45$ years [16]. In addition, a study on medullablastomas reported TERT mutations to be significantly more frequent in an older cohort of pediatric patients (median age: 16 years) compared to a very young patient cohort (median age: 6 years) [17]. All these data gave evidence for a minor role of TERT promoter mutation in tumors from patients with early-onset disease.

It is difficult to explain this age-dependency. Focusing on bladder cancer it was recently shown that TERT promoter mutations lead to increased levels of TERT mRNA, TERT protein, telomerase enzymatic activity and telomere length in vitro [18]. Moreover, increased levels of TERT mRNA were shown to be associated with a reduced disease-specific survival suggesting high levels of TERT mRNA as a marker for biological aggressiveness in bladder cancer [18]. Our findings of a low TERT promoter mutation frequency in bladder tumors from young patients fits well with this observation as bladder tumors in young patients showed a low biological aggressiveness, and patients with early-onset disease displayed a favorable course of disease in most cases investigated $[19,20]$.

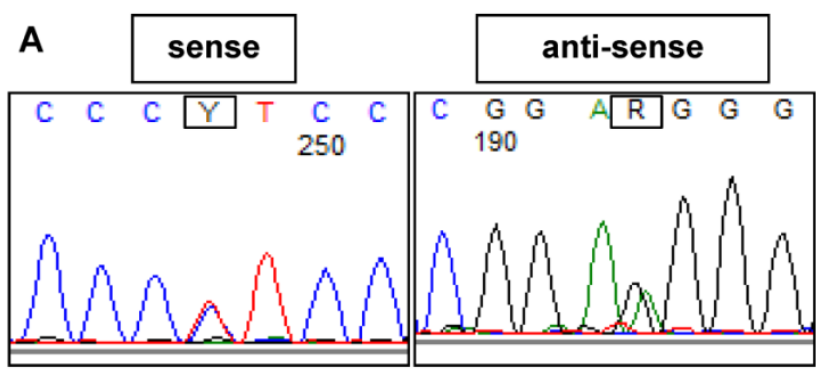

B

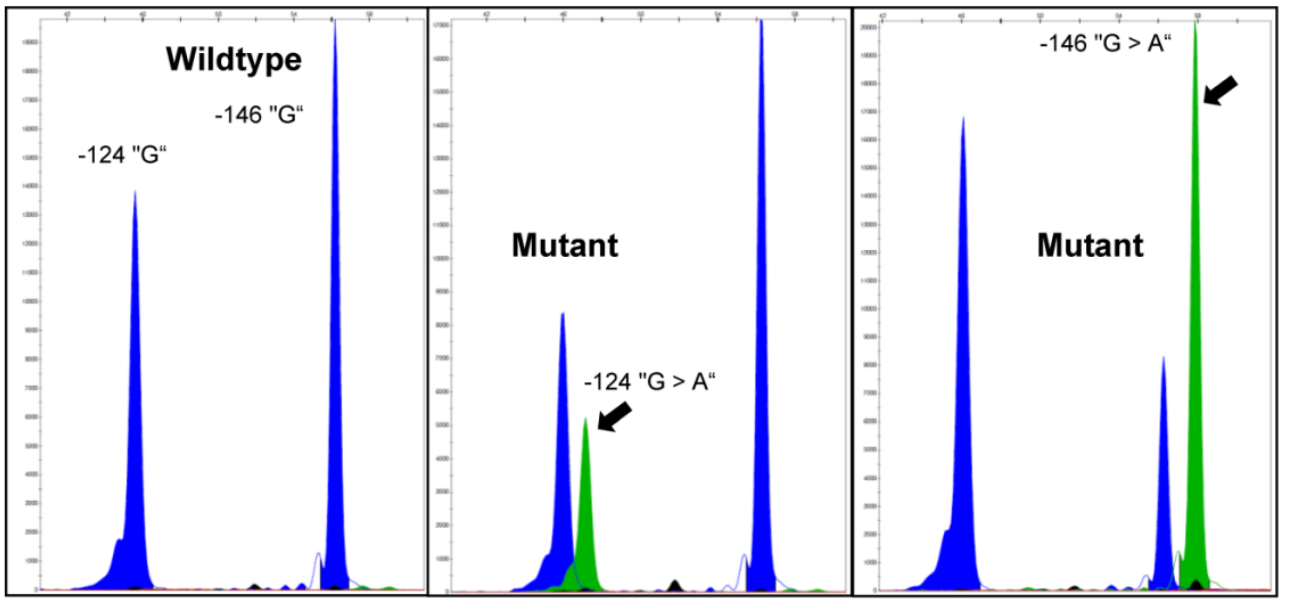

Figure 2: Representative examples of TERT promoter mutations detected in bladder tumors using Sanger sequencing or SNaPshot analysis. A: Electropherograms of Sanger sequencing results for a -124 "G > A" mutant sample. B: SNaPshot profiles for wildtype, -124 "G > A" mutant and -146 "G > A" mutant samples. Arrows indicate mutant peaks. 


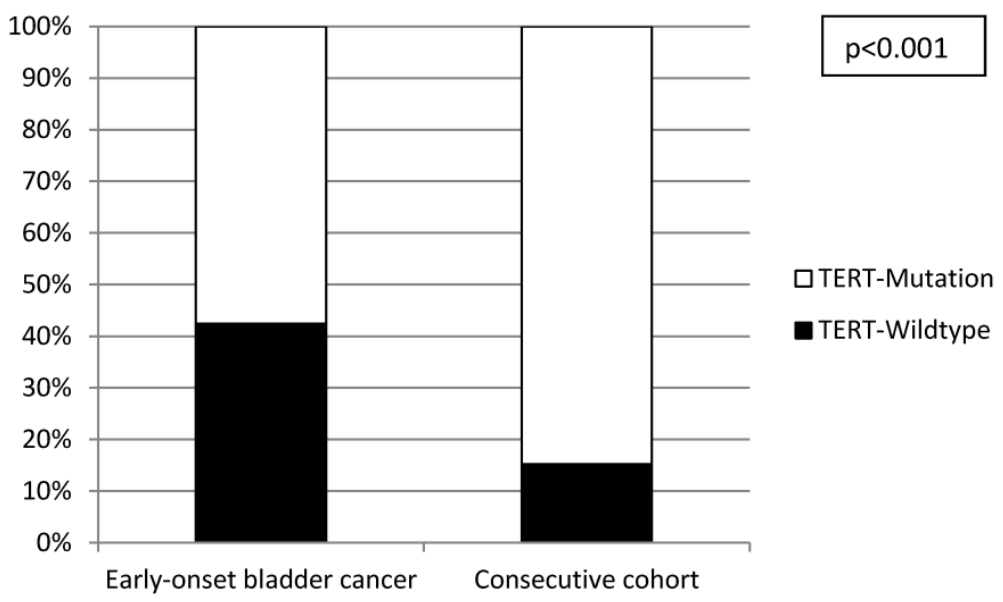

Figure 3: Distribution of TERT promoter mutations in the early-onset and consecutive bladder cancer cohorts.

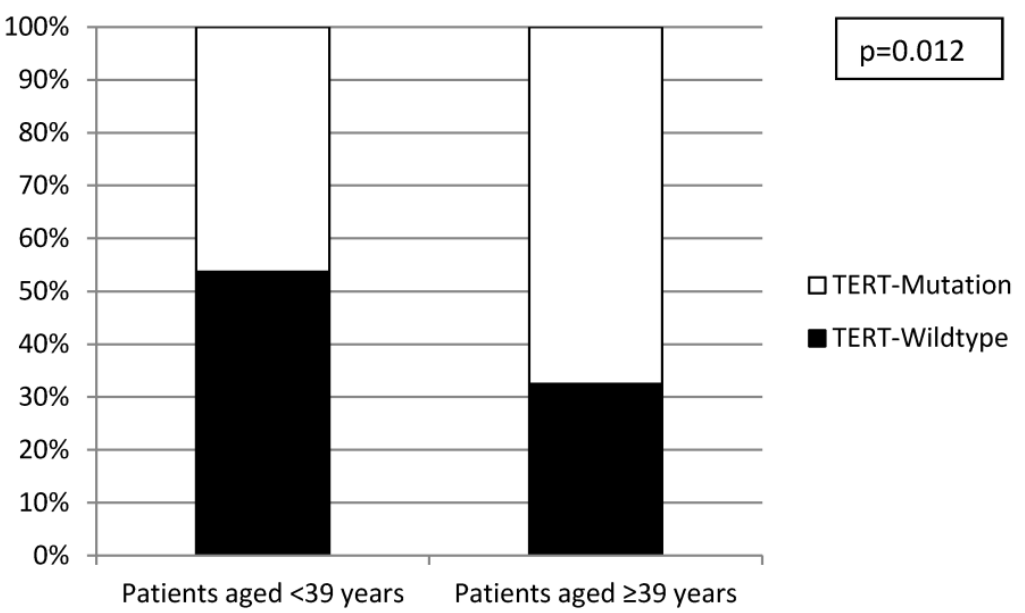

Figure 4: Distribution of TERT promoter mutations within the early-onset bladder cancer cohort stratified according to median age.

TERT promoter mutations were described as being frequently concomitant with FGFR3 mutations in bladder tumors especially in tumors with low grade and low stage [21]. Based on the limited molecular data available on early-onset bladder tumors FGFR3 mutation is a rare event in bladder tumors from young adults [22, 23]. As FGFR3 and TERT promoter mutations are the most frequent alterations in bladder cancer the low frequency of both mutations in tumors from patients with early-onset disease argues for a specific and discrete molecular background of tumorigenesis in these patients. In addition, in $4.2 \%$ of the tumors from the early-onset tumor cohort we detected a double mutation at positions -124 and -146 (Table 2). In contrast, in our consecutive cohort as well as in published cohorts mutations at these positions were found as being mutually exclusive [4, 5]. One explanation for this finding might be a possible molecular heterogeneity of the tumors, i.e. two separate clones within the sample each with a different mutation. A second explanation might be an acquired second mutation during the progress of malignant transformation. This second TERT mutation might be a rare event and possibly speed up a carcinogenic process. As a methodological proof of this concept is difficult this suggestion remains speculative but would be in favor of an exclusive molecular feature of tumors from young patients. Our own studies on epigenetic changes in bladder tumors underlined a discrete molecular background in these cases as patients below 19 years of age showed a low rate of epigenetic alterations compared to elderly patients [24].

This first report on TERT promoter mutations in bladder tumors from a large cohort of patients with early-onset disease has shown that these tumors have a lower mutation frequency as compared to that reported in patients with regular disease-onset. These data further strengthen the suggestion of a distinct 
molecular tumorigenesis in young bladder cancer patients.

\section{Acknowledgment}

The authors thank Karina Kalb, Stefanie Herlein, Claudia Schmied, Nina Oks and Verena Popp for excellent technical assistance. This study was supported by a grant of the ELAN Fonds of the University of Erlangen to JG. We acknowledge support by Deutsche Forschungsgemeinschaft and Friedrich-Alexander-Universität Erlangen-Nürnberg (FAU) within the funding programme Open Access Publishing.

\section{Competing Interests}

The authors have declared that no competing interest exists.

\section{References}

1. Low KC, Tergaonkar V. Telomerase: central regulator of all of the hallmarks of cancer. Trends in biochemical sciences. 2013; 38: 426-34

2. Horn S, Figl A, Rachakonda PS, Fischer C, Sucker A, Gast A, et al. TERT promoter mutations in familial and sporadic melanoma. Science. 2013; 339: 959-61.

3. Vinagre J, Pinto V, Celestino R, Reis M, Populo $\mathrm{H}$, Boaventura $\mathrm{P}$, et al Telomerase promoter mutations in cancer: an emerging molecular biomarker? Virchows Archiv : an international journal of pathology. 2014; 465: 119-33.

4. Hurst CD, Platt FM, Knowles MA. Comprehensive mutation analysis of the TERT promoter in bladder cancer and detection of mutations in voided urine. European urology. 2014; 65: 367-9.

5. Allory Y, Beukers W, Sagrera A, Flandez M, Marques M, Marquez M, et al. Telomerase reverse transcriptase promoter mutations in bladder cancer: high frequency across stages, detection in urine, and lack of association with outcome. European urology. 2014; 65: 360-6.

6. Wu S, Huang $\mathrm{P}, \mathrm{Li} C$, Huang $Y, \mathrm{Li} X$, Wang $Y$, et al. Telomerase reverse transcriptase gene promoter mutations help discern the origin of urogenital tumors: a genomic and molecular study. European urology. 2014; 65: 274-7.

7. Kinde I, Munari E, Faraj SF, Hruban RH, Schoenberg M, Bivalacqua T, et al. TERT promoter mutations occur early in urothelial neoplasia and are biomarkers of early disease and disease recurrence in urine. Cancer research. 2013; 73: 7162-7.

8. Cheng L, Davidson DD, Wang M, Lopez-Beltran A, Montironi R, Wang L, et al. Telomerase reverse transcriptase (TERT) promoter mutation analysis of benign, malignant and reactive urothelial lesions reveals a subpopulation of inverted papilloma with immortalizing genetic change. Histopathology. 2015.

9. Zheng X, Zhuge J, Bezerra SM, Faraj SF, Munari E, Fallon JT, 3rd, et al. High frequency of TERT promoter mutation in small cell carcinoma of bladder, but not in small cell carcinoma of other origins. Journal of hematology \& oncology. 2014; $7: 47$.

10. Paner GP, Zehnder P, Amin AM, Husain AN, Desai MM. Urothelial neoplasms of the urinary bladder occurring in young adult and pediatric patients: a comprehensive review of literature with implications for patient management. Advances in anatomic pathology. 2011; 18: 79-89.

11. Mostofi FK SL, Torloni H. International Histological Classification of Tumours. Geneva: World Health Organization; 1973.

12. Sobin LH WC. TNM Classification of Malignant Tumors (6th edn). New York: John Wiley \& Sons: Hoboken; 2002.

13. Stoehr R, Zietz S, Burger M, Filbeck T, Denzinger S, Obermann EC, et al. Deletions of chromosomes 9 and $8 \mathrm{p}$ in histologically normal urothelium of patients with bladder cancer. European urology. 2005; 47: 58-63.

14. Stoehr R, Taubert H, Zinnall U, Giedl J, Gaisa NT, Burger M, et al. Frequency of TERT Promoter Mutations in Prostate Cancer. Pathobiology : journal of immunopathology, molecular and cellular biology. 2015; 82: 53-7.

15. Wang K, Liu T, Ge N, Liu L, Yuan X, Liu J, et al. TERT promoter mutations are associated with distant metastases in upper tract urothelial carcinomas and serve as urinary biomarkers detected by a sensitive castPCR. Oncotarget. 2014; 5: 12428-39.

16. Liu T, Wang N, Cao J, Sofiadis A, Dinets A, Zedenius J, et al. The age- and shorter telomere-dependent TERT promoter mutation in follicular thyroid cell-derived carcinomas. Oncogene. 2014; 33: 4978-84.

17. Killela PJ, Reitman ZJ, Jiao Y, Bettegowda C, Agrawal N, Diaz LA, Jr., et al. TERT promoter mutations occur frequently in gliomas and a subset of tumors derived from cells with low rates of self-renewal. Proceedings of the National Academy of Sciences of the United States of America. 2013; 110: 6021-6.
18. Borah S, Xi L, Zaug AJ, Powell NM, Dancik GM, Cohen SB, et al. Cancer. TERT promoter mutations and telomerase reactivation in urothelial cancer. Science. 2015; 347: 1006-10.

19. Comperat E, Larre S, Roupret M, Neuzillet Y, Pignot G, Quintens H, et al. Clinicopathological characteristics of urothelial bladder cancer in patients less than 40 years old. Virchows Archiv : an international journal of pathology. 2015; 466: 589-94

20. Wang QH, Ji ZG, Li HZ, Fan H, Chen ZG, Shi BB, et al. Clinicopathologic Comparison of Urothelial Bladder Carcinoma in Young and Elder Patients. Pathology oncology research : POR. 2015.

21. Hosen I, Rachakonda PS, Heidenreich B, de Verdier PJ, Ryk C, Steineck G, et al. Mutations in TERT promoter and FGFR3 and telomere length in bladder cancer. International journal of cancer Journal international du cancer. 2015; 137: 1621-9.

22. Wild PJ, Giedl J, Stoehr R, Junker K, Boehm S, van Oers JM, et al. Genomic aberrations are rare in urothelial neoplasms of patients 19 years or younger. The Journal of pathology. 2007; 211: 18-25.

23. Williamson SR, Wang M, Montironi R, Eble JN, Lopez-Beltran A, Zhang S, et al. Molecular characteristics of urothelial neoplasms in children and young adults: a subset of tumors from young patients harbors chromosomal abnormalities but not FGFR3 or TP53 gene mutations. Modern pathology : an official journal of the United States and Canadian Academy of Pathology, Inc. 2014; 27: 1540-8.

24. Owen HC, Giedl J, Wild PJ, Fine SW, Humphrey PA, Dehner LP, et al. Low frequency of epigenetic events in urothelial tumors in young patients. The Journal of urology. 2010; 184: 459-63. 